\title{
Elucidation of PEGylation Site with a Combined Approach of In-Source Fragmentation and CID MS/MS
}

\author{
Xiaojun Lu, P. Clayton Gough, Michael R. DeFelippis, and Lihua Huang \\ Bioproduct Research and Development, Lilly Research Laboratories, Eli Lilly and Company, Lilly Corporate \\ Center, Indianapolis, Indiana, USA
}

Poly(ethylene glycol) (PEG)ylation of peptides and proteins creates significant challenges for detailed structural characterization, such as PEG heterogeneity, site of addition and number of attached PEGylated moieties. Recently, we published a novel LC/MS methodology with a post-column addition of amines to obtain accurate masses of PEGylated peptides and proteins. The accurate masses can be used to assign the structures and number of attached PEGs [15], but the PEGylation site remains unclear in situations where multiple potential attachments are involved. Here, we present a methodology combining in-source fragmentation (ISF) with CID-MS/MS to elucidate the PEGylated sites in PEGylated products. All PEGylated samples, either prepared in acidic solution, or collected from a RP-HPLC stream, were first ionized via ISF to produce products containing small PEG fragment attachment, and then those fragment ions obtained were sequenced via CID MS/MS to deduce the PEGylation site. The methodology was successfully applied to PEGylated glucagon and IgG4 antibody light chain, which demonstrated that the small PEG fragments attached were stable during the CID activation. (J Am Soc Mass Spectrom 2010, 21, 810-818) (c) 2010 American Society for Mass Spectrometry

$\mathrm{P}$ roteins and peptides have experienced tremendous growth in the pharmaceutical industry as potential therapeutics since the inception of recombinant DNA technologies and advances in solidphase synthetic methods [1,2]. Despite these advances in production capability, the inherent chemical and physical instability of peptides and proteins as well as their susceptibility to proteolytic degradation and rapid clearance in vivo complicates formulation development and limits delivery to daily injections. Because injections are considered inconvenient and may impact patient compliance, one approach has focused on increasing the short circulating half-life of peptides and proteins in vivo (pharmacokinetics) by means of molecular modifications. Common molecular modification strategies include: increasing the sialic acid content of glycan residues [3], covalent conjugation with albumin $[4,5]$, or the Fc region of IgG [6], and most successfully, attachment of water-soluble poly(ethylene glycol) (PEG) moieties [7].

Following the pioneering research of Davis [7-8] and Abuchowsky [7] in late 1970s, PEGylation of peptides and proteins has quickly become a widely used drug

Address reprint requests to Dr. L. Huang, Lilly Research Laboratories, Eli Lilly and Company, Indianapolis, IN 46285, USA. E-mail: huang_lihua@lilly.com This work was initially presented as a poster at the 57th ASMS Conference on Mass Spectrometry and Allied Topics on May 31-June 4, 2009, in Philadelphia, PA. enhancement approach. Addition of non-toxic, nonimmunogenic, and amphiphilic PEG moieties should not impair the efficacy of biopharmaceuticals but significantly improve the pharmacokinetic behavior by increasing solubility, reducing immunogenicity, improving stability, and extending circulation half-life in vivo [9-11]. Since the first PEGylated biopharmaceutical, 'Adagen', was approved by the FDA in 1990, there have been at least eight PEGylated proteins approved and marketed, and still more in clinical trials, which target a broad range of diseases including cancer, hepatitis, gout, and diabetes [11]. Despite the growing interest, PEGylation of peptides and proteins still creates significant challenges for detailed structural characterization, such as the PEG polydispersity, site of addition, and number of attached PEGs.

When conventional ESI-MS was used for detection, PEGylated products were normally heavily charged, leading to overlap of different charge ions in the final mass spectra and often the fragmentation of the PEG chains. As a result of these issues, most previously reported studies relied on MALDI-MS due to its typical singly-charged ionization [12-14]. Nevertheless, the application of MALDI to large molecular weight PEGylated products often yielded limited structural information due to the technique's limited resolution in the linear mode. Recently, we published a novel LC/ESI-MS methodology with a post-column addition of amines to obtain accurate masses of PEGylated peptides and 
proteins [15]. While the accurate masses can be used to assign some structural elements (e.g., dimerization, oxidation, maleimide ring opening by hydrolysis, etc.) and number of attached PEGs, the site of PEG addition still remained unknown in situations where multiple potential sites existed.

Depending on the proximity to the binding region, PEGylated sites might have an influence on the biological activity of the conjugated molecules. Furthermore, the presence of multiple potential PEGylation sites could easily lead to a mixture of positional isomers, so the identification of those sites is very crucial for the characterization of PEGylated drugs, and provides important information needed to support regulatory submissions. To date, many techniques have been combined to address the problem of locating PEG attachment sites [16-25]. Edman degradation, the most widely used method for short peptides, was applied to determine the PEGylation site based on the absence of expected amino acids in the sequence [19]. Since large peptides and proteins often require prior enzyme cleavage to generate smaller fragments, the application of Edman degradation has to overcome the difficulties in isolating and purifying the individual fragments after digestion. Some studies, based on enzymatic digestion followed by peptide mapping, reported that the PEGylation sites could be located by comparing the peptide maps of PEGylated and native proteins [20], but the PEGylation itself might also affect the protease digestion and, therefore, complicate the final data interpretation. All of the cited studies focused on samples modified with relatively small size PEGs. Recently, a novel MALDI-TOF methodology using reflector insource decay successfully identified the PEGylation site of $20 \mathrm{kDa}$ PEGylated peptides because the attachment of the large PEG truncated the fragment ion series at the PEGylation site (residue) [18]. However, this method is not applicable when multiple potential sites exist. Another conventional method involves dePEGylation, which removes PEG moieties from the protein molecule and minimizes the difficulties of PEGylation site mapping. For example, BrCN treatment of two novel PEG derivatives, PEG-Met-Nle-OSu and PEG-Met- $\beta$ AlaOSu, removed PEG leaving Nle or $\beta$ Ala at the original PEGylated sites [21]. NMR is another powerful tool that has been applied for PEGylation site mapping [24, 25], but the general limitation associated with analysis of large molecular weight samples still must be considered. Taken together, current PEGylation site mapping procedures are either based on indirect observations, or only limited to specific PEGylated molecules. Here we report a novel PEGylation site mapping procedure that is based on direct observation from conventional CID MS/MS for peptide ions with small PEG fragment attached, which was obtained during electrospray ionization with in-source fragmentation.

\section{Experimental}

\section{Materials}

Intact glucagon, $5 \mathrm{kDa}$ PEGylated glucagon, glucagon, and IgG4 antibody were produced at Eli Lilly and Company (Indianapolis, IN, USA); $20 \mathrm{kDa}$ methoxyl poly(ethylene glycol) para-nitrophenylcarbonate (mPEGPNP) was purchased from Dow Chemical (Midland, MI, USA); $20 \mathrm{kDa}$ methoxyl poly(ethylene glycol), maleimide (mPEG-MAL) was purchased from NOF Corporation (Tokyo, Japan). All standard buffer solutions were purchased from Fisher Scientific (Pittsburgh, PA, USA). Diethylmethylamine (DEMA) was purchased from Aldrich (St. Louis, MO, USA). Endoproteinase Lys-C was purchased from Wako Chemical USA, Inc. (Richmond, VA, USA). Dithiothreitol (DTT) and $8.0 \mathrm{M}$ Guanidine $\mathrm{HCl}$ were purchased from Pierce Biotechnology (Rockford, IL, USA). All other chemicals were reagent grade and commercially available.

\section{Sample Preparation}

To demonstrate the application of the methodology, two model PEGylated products, PEGylated glucagon and partially reduced IgG light chain were used. A variety of PEGylation chemistries is available to attach poly(ethylene glycol) (PEG) to peptide or protein (PEGylation) [26], and two approaches were employed in this work. A 5 or $20 \mathrm{kDa}$ PEGylated glucagon sample was made by reacting glucagon with 5 or $20 \mathrm{kDa}$ methoxyl poly(ethylene glycol) para-nitrophenylcarbonate (mPEG-PNP), which reacts with amine groups of Lys and His or the N-terminus. Glucagon has two potential reaction sites, His1 and Lys12. A $20 \mathrm{kDa}$ PEGylated IgG4 light chain sample was synthesized through maleimide chemistry using $20 \mathrm{kDa}$ methoxyl poly(ethylene glycol) maleimide (mPEG-MAL) reaction with sulfhydryl group of Cys residue of partially reduced IgG4 light chain. Partially reduced IgG4 light chain only contains one reduced Cys residue.

The $20 \mathrm{kDa}$ PEGylated glucagon and $20 \mathrm{kDa}$ PEGylated IgG4 antibody samples were prepared as previously described [15]. A solution of $5 \mathrm{kDa}$ PEGylated glucagon was freshly prepared at a concentration of $0.5 \mathrm{mg} / \mathrm{mL}$ in $0.05 \%$ TFA before MS analysis. To produce positional isomers having alternate sites of PEGylation, different $\mathrm{pH}$ buffer solutions were employed to adjust the synthetic conditions for PEGylation. To locate the conjugation site of PEGylated IgG4 before the Lys-C digestion, PEGylated IgG4 antibody was subjected to full reduction and alkylation using DTT and iodoacetamide, respectively. Briefly, 200 $\mu \mathrm{L}$ of PEGylated IgG4 solution ( $~ 100 \mu \mathrm{g}$ antibody) was lyophilized and resuspended in $10 \mu \mathrm{L}$ of $250 \mathrm{mM}$ Tris buffer, $\mathrm{pH} 8$, containing $6 \mathrm{M}$ guanidine $\cdot \mathrm{HCl}$. After the sample was fully dissolved, the solution was mixed with $2 \mu \mathrm{L}$ of $500 \mathrm{mM}$ DTT with subsequent incubation 
at $37^{\circ} \mathrm{C}$ for $1 \mathrm{~h}$. Fully reduced PEGylated IgG4 antibody was alkylated by adding $5 \mu \mathrm{L}$ of $50 \mathrm{mg} / \mathrm{mL}$ iodoacetamide and then diluted to $100 \mu \mathrm{L}$ with $50 \mathrm{mM}$ Tris buffer, $\mathrm{pH}$ 8.0. The final sample was mixed with Lys-C in a 1:50 enzyme-to-antibody ratio (weight/weight) and incubated at $37^{\circ} \mathrm{C}$ for $3 \mathrm{~h}$.

\section{Reversed-Phase HPLC Chromatography}

A Waters Alliance HPLC system model 2795 or Acquity UPLC (Waters, Milford, MA, USA) was used for all the separations. For all analyses, the HPLC column was a Polymer Lab polymer C18 $(2.1 \times 50 \mathrm{~mm}, 8 \mu \mathrm{m}$ particle size, $1000 \AA$ pore size) (Amberst, MA, USA). The flow rate was $200 \mu \mathrm{L} / \mathrm{min}$, and the column was maintained at $60{ }^{\circ} \mathrm{C}$. The solvents were: (A) $0.05 \%$ aqueous trifluoro acetic acid (TFA) solution, and (B) $0.04 \%$ TFA in acetonitrile. For intact PEGylated glucagon, partially reduced IgG4 or Lys-C digest analysis, the column was equilibrated with $20 \%$ B before sample injection. After the injection of sample, a gradient elution was performed from $20 \%$ to $40 \%$ B in $8 \mathrm{~min}$, to $52 \%$ (46\% for UPLC) B over $2 \mathrm{~min}$, to $56 \% \mathrm{~B}$ (49\% for UPLC) over $14 \mathrm{~min}$, then to $90 \%$ B over $1 \mathrm{~min}$, and held at $90 \%$ B for $1.5 \mathrm{~min}$, and then rapidly returned to initial conditions. Post-column addition was performed with a steady flow rate of 60 $\mu \mathrm{L} / \mathrm{min}$ of amine solution $(0.05 \%$ amine in $50 \%$ acetonitrile solution) introduced by HPLC pump.

\section{MS Analysis}

A Waters Alliance HPLC or Acquity UPLC system model 2795 was coupled to a Waters LCT Premier or a Waters SYNAPT HDMS mass spectrometer (Waters Corp.). The HPLC effluent was introduced directly into the mass spectrometer without splitting the stream. The samples were first characterized using LC-MS with a post-column addition of DEMA as previously described [15]. MS was performed with the following settings: capillary voltage of $3.2 \mathrm{kV}$, cone voltage of $150 \mathrm{~V}$, Aperture 1 of $100 \mathrm{~V}$, mass range of 400 to 15,990 , desolvation temperature of $300{ }^{\circ} \mathrm{C}$, and desolvation gas flow at $700 \mathrm{~L} / \mathrm{h}$. Deconvolution of collected mass spectra was carried out using MassLynx (ver. 4.1) MaxEnt 1 software (Waters Corp.). To induce ISF of PEGylated products, the same LC-MS method was performed without the post-column addition of DEMA, and either aperture 1 (LCT Premier) or cone voltage (SYNAPT) of mass spectrometer was elevated to obtain protein or peptide attached with small PEG fragments. MS was performed with the following settings: capillary voltage of $3.2 \mathrm{kV}$, cone voltage of 150 or $60 \mathrm{~V}$ (for SYNAPT), Aperture 1 of 60 or $0 \mathrm{~V}$ (for SYNAPT), mass range of $400-15,990$ or $50-2000$ or 3000 (for SYNAPT), desolvation temperature of $300{ }^{\circ} \mathrm{C}$, and desolvation gas flow at $700 \mathrm{~L} / \mathrm{h}$. CID MS/MS measurements were performed using a Waters SYNAPT HDMS mass spectrometer, where fragment ions of the peptides attached with small PEG fragments were selectively activated by CID (collision energy of 50.0 or $25 \mathrm{eV}$ for PEGylated glucogan or Lys-C peptide of IgG4 light chain, respectively) and then analyzed in the TOF analyzer. The MS/MS spectrum for $5 \mathrm{kDa}$ PEGylated glucagon was obtained with the direct infusion at $10 \mu \mathrm{L} / \mathrm{min}$.

\section{Results and Discussion}

DePEGylation is an efficient approach to simplify the PEGylated products and enable determination of the PEGylation site. However, previous established dePEGylation procedures are only applicable for certain PEG linkages, and often require specific reagents. As mentioned, the application of conventional ESI-MS for PEGylation characterization normally leads to the fragmentation of PEG chains, a process that is similar to dePEGylation but can be generally employed for a variety of PEGylated products. Here, we utilized charge-induced fragmentation to facilitate the identification of PEGylation site.

\section{Five kDa PEGylated Glucagon}

Five kDa PEGylated glucagon, whose PEGylation site had been mapped to the residue Lys12 by previous electron transfer dissociation (ETD) experiments [27], was first selected as a model system for our studies. As shown in Figure 1a, with a post-column addition of DEMA, deconvoluted mass spectra clearly revealed the polydispersity of amine-adducted molecules. It should be noted that the deconvoluted MS spectrum does not have an expected Gaussian distribution for the PEGylated glucagon sample. The cause of this profile is likely related to the manner in which the sample was purified. The mass spectral (molecular weight) profile of PEG or PEGylated product should have a normal (Gaussian) distribution. However, the $5 \mathrm{kD}$ PEGylated glucagon sample we used did not show a normal distribution, and the high mass region was truncated (see Figure 1). This is because the $5 \mathrm{kDa}$ PEGylated glucagon used in our study was a purified product by RP-HPLC after the PEGylation. To obtain purer PEGylated glucagon, the late eluting region of the PEGylated glucagon peak was cut during the peak collection. To induce the ISF, we removed the postcolumn addition of DEMA, and treated the $5 \mathrm{kDa}$ PEGylated glucagon sample with harsh mass spectrometric conditions (i.e., high aperture 1 or sample cone voltages). As shown in Figure $1 b$, a series of triply charged ions were observed in the 1300-2000 ( $\mathrm{m} / \mathrm{z})$ range with a 14.7 spacing between each adjacent ion peak. Since short PEG fragmentation ions are normally singly charged based on the same analysis for PEG itself [15], these ions should represent a series of PEGylated glucagon with a mass range of 3900-6000 Da. Because the molecular weight of each oxyethylene unit is $44 \mathrm{Da}$, the number of oxyethylene units can be easily calculated by the accurate mass for each ion. For example, 


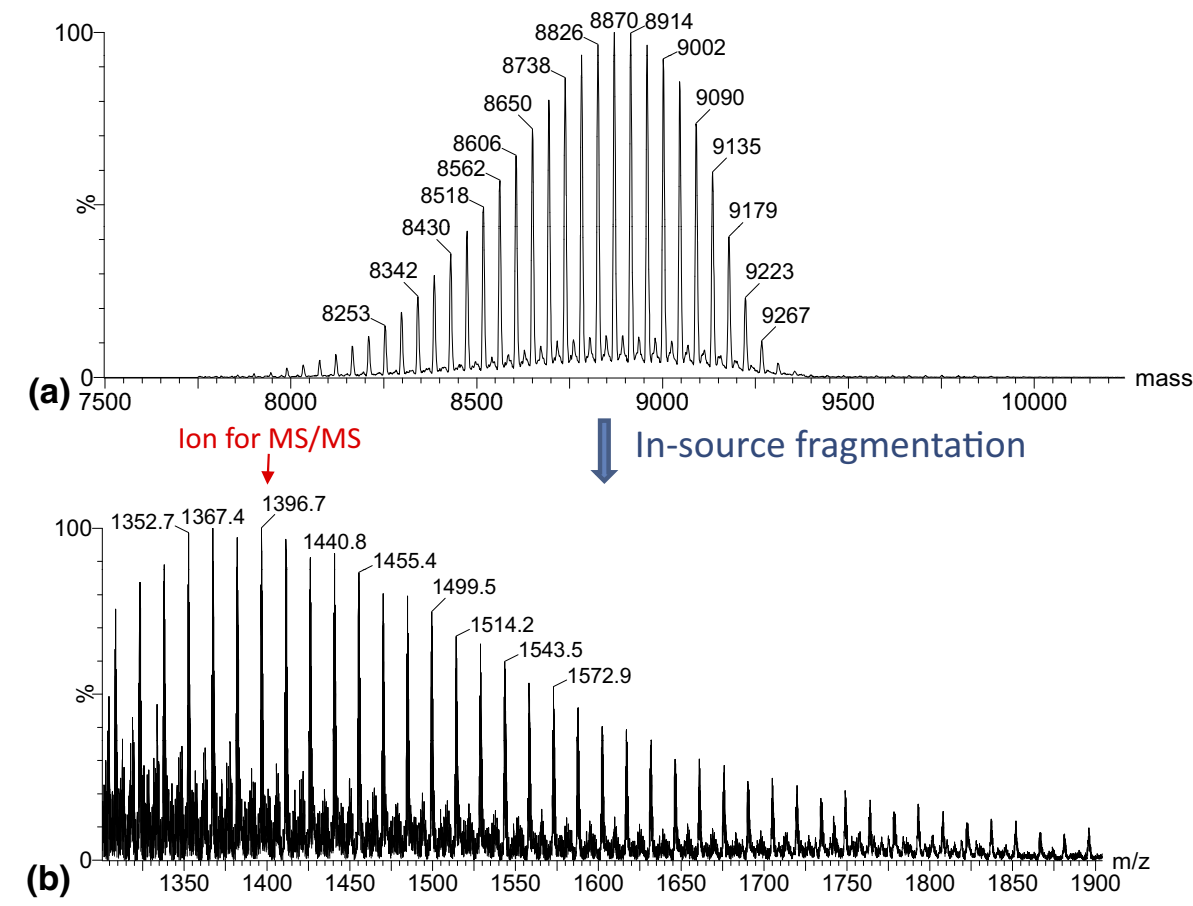

Figure 1. Deconvoluted mass spectrum (a) of $5 \mathrm{kDa}$ PEGylated glucagon with a post-column addition of DEMA, and mass spectrum (b) of $5 \mathrm{kDa}$ PEGylated glucagon after ISF. The precursor ions selected for the subsequent CID MS/MS analysis is marked by the red arrow.

the triply charged ion at $\mathrm{m} / \mathrm{z} 1396.7$ (1396.7 is the strongest isotopic ion and the monoisotopic ion is 1396.0) is highly consistent with the expected mass of
4185.0 Da for truncated PEGylated glucagon containing a $44 \mathrm{Da}$ linkage and 15 oxyethylene units (glucagon + $\mathrm{C} 31 \mathrm{H} 60 \mathrm{O} 17=3480.6156+704.3831=4185.0 \mathrm{Da})$.

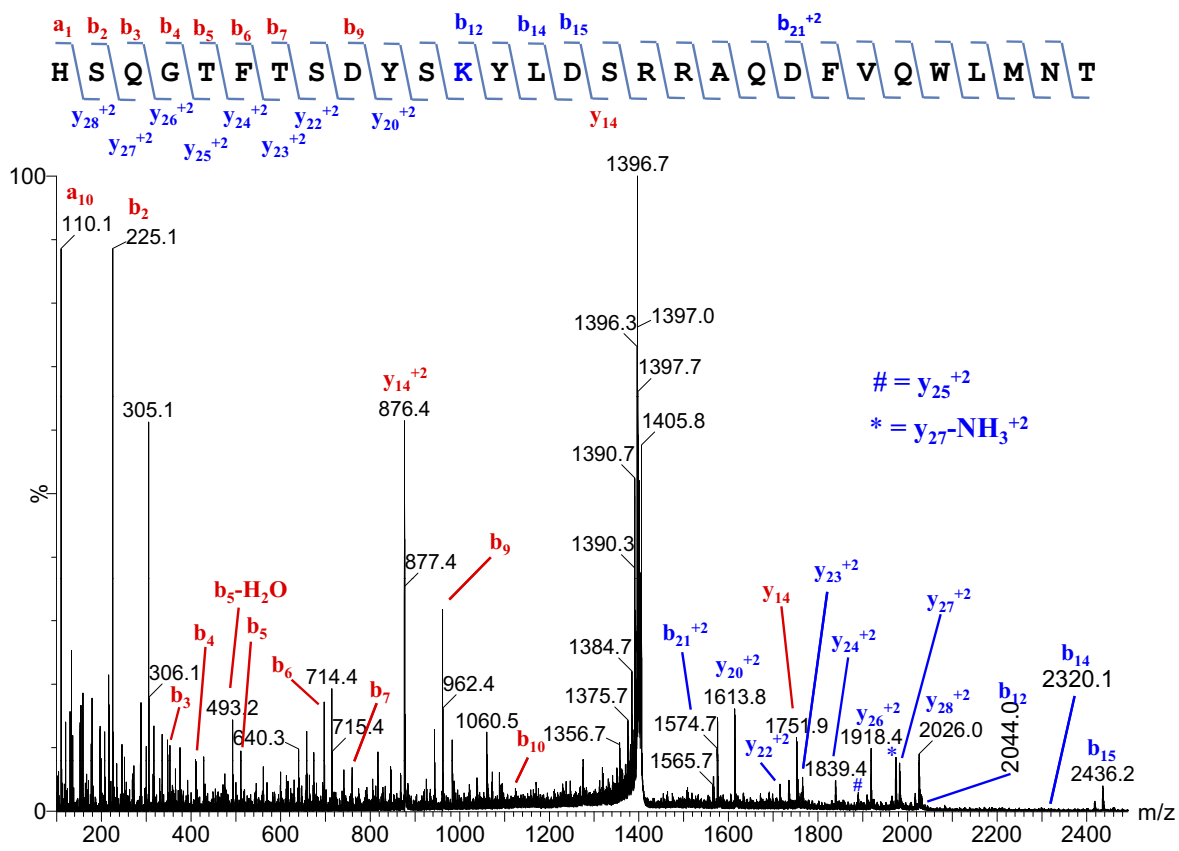

Figure 2. MS/MS spectrum of $5 \mathrm{kDa}$ PEGylated glucagon after ISF. The $\mathrm{m} / \mathrm{z}(\mathrm{z}=3)$ of the precursor ion is 1396.7 (glucagon + a small PEG fragment), and the interpretation of this product ion spectrum is summarized at the top of the spectrum (the detailed MS/MS data assignment is provided in Table 1 in the supplemental materials). The red and blue labels represent the ions without and with PEG attachment, respectively. 
To localize the PEGylation site, the ion at $m / z 1396.7$ was selected as the precursor ion to perform conventional CID MS/MS. As shown in Figure 2, all b ions that are smaller than the $b_{11}$ ion were found unmodified, but the singly charged $b_{15}$ ion shifted from 1730.8 to 2435.3 , and the doubly charged $b_{21}$ ion also shifted from 1222.6 to 1574.8. The mass shifts from both $b$ ions are equal and match exactly the short PEG attachment containing 15 oxyethylene units. Similarly, all y ions containing residue Lys12 are also found to be PEGylated with 15 oxyethylene units, while those without the attachment have the expected calculated mass, indicating no PEG attachment. Taken together, the combination of ISF and CID MS/MS allowed us to directly determine that glucagon was PEGylated at Lys12, which was consistent with previous results. Nevertheless, compared with previous studies that relied on the high-end ETD instrumentation, our methodology only employed conventional CID MS/MS and can be utilized in a wider range of labs.

The data for PEGylated glucagon revealed that depending on the size of PEG chain, the molecule might have two distinct stabilities in a mass spectrometer.
During ISF, the PEG chains of $5 \mathrm{kDa}$ PEGylated glucagon were unstable and quickly dissociated while the peptide bonds of glucagon had been less affected. During CID MS/MS, those low molecular weight PEG fragments truncated by ISF were preserved while, instead, the peptide bonds were dissociated. This observation might result from the different charge distribution of long and short PEG chains conjugated to a peptide/protein. During the ionization, the PEG chains of $5 \mathrm{kDa}$ PEGylated glucagon are heavily charged. The PEG ether bond with $\mathrm{H}^{+}$ion is labile and completely cleaved under ISF conditions. In contrast, the truncated PEG chains obtained in the experiment do not carry any charges $\left(\mathrm{H}^{+}\right)$. Thus, the attached small PEG fragment was retained during the dissociation of polypeptide chains.

\section{Twenty kDa PEGylated Glucagon}

It has been known that the size of PEG has to be at least $20 \mathrm{kDa}$ to retard renal clearance effectively [28]. Although the ETD approach can be used to directly determine the PEGylation site of $5 \mathrm{kDa}$ PEGylated

(a)

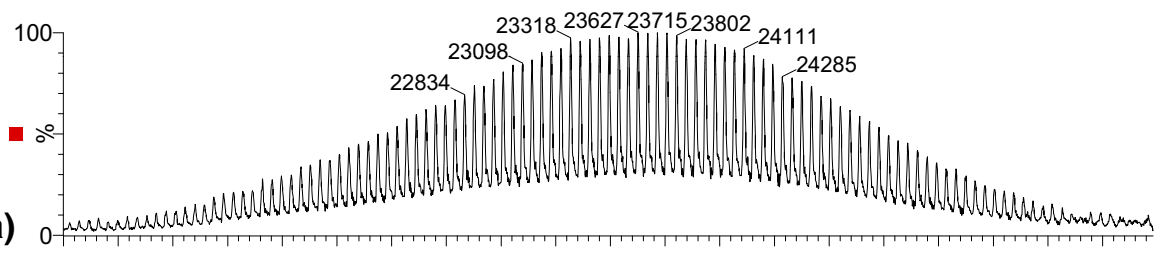

(b)

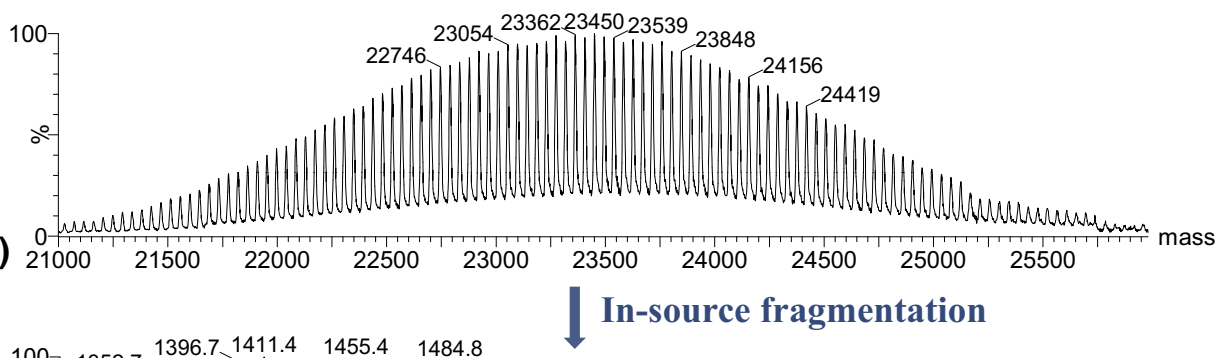

(c)
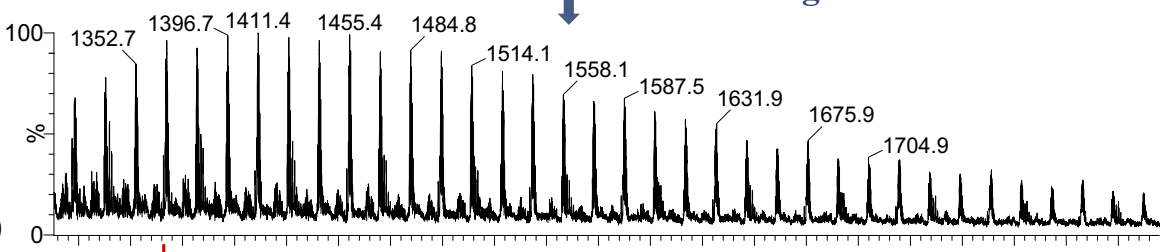

(d)

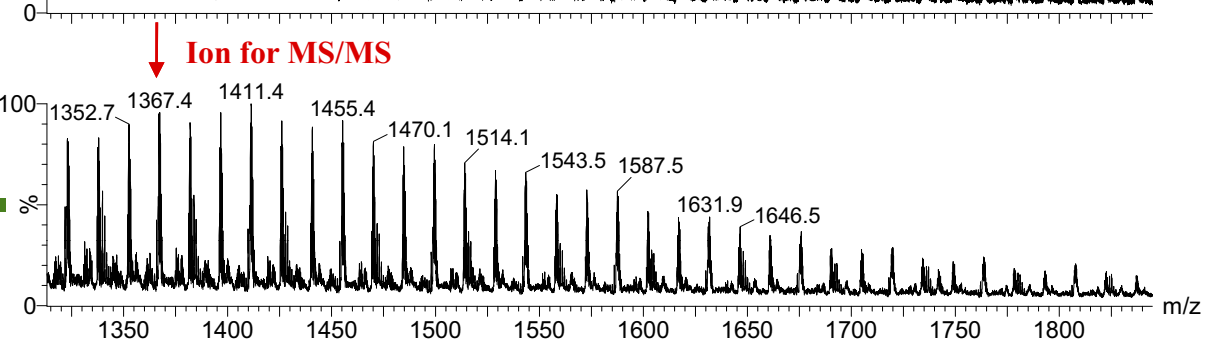

Figure 3. Deconvoluted mass spectra of $20 \mathrm{kDa}$ PEGylated glucagon prepared at pH10 (a) and pH7 (b) with a post-column addition of DEMA, and mass spectra of $20 \mathrm{kDa}$ PEGylated glucagon prepared at $\mathrm{pH} 10$ (c) and $\mathrm{pH} 7$ (d) after ISF. 
glucagon, the same procedure is impractical for $20 \mathrm{kDa}$ PEGylated glucagon. Our methodology, where the PEGylated products are initially fragmented by ISF, should not have the same size limitation as the ETD approach. To evaluate the capability of our methodology, 20 kDa PEGylated glucagon was prepared by incubating glucagon and $20 \mathrm{kDa}$ mPEG-PNP together at $\mathrm{pH}$ 10. Following analysis of the sample using the post-column addition of amine method, the deconvoluted mass spectra revealed that the molecular weight of DEMA-adducted mono-PEGylated glucagon has a Gaussian distribution in the 21,000 to $26,000 \mathrm{Da}$ range (Figure 3a). As shown in Figure 3c, the ISF treatment of these molecules led to a similar fragment ion pattern as obtained for $5 \mathrm{kDa}$ PEGylated glucagon. The following CID MS/MS analysis of the ion at $\mathrm{m} / \mathrm{z} 1367.3$ (monoisotopic mass ion is 1366.7) successfully confirmed that the $20 \mathrm{kDa}$ PEGylated glucagon we prepared is also PEGylated at the Lys12 residue (see the next paragraph). Thus, our methodology is not limited by the size of PEG.

Glucagon contains two primary amine groups that can react with $\mathrm{MPEG}-\mathrm{PNP}$; one at the side chain of the Lys12 residue and the other at the $\mathrm{N}$-terminus. Due to different $\mathrm{pKa}$ values at these locations, only the lysine amine is PEGylated at $\mathrm{pH}$ 10. PEGylation at the $\mathrm{N}$ terminus normally occurs at lower $\mathrm{pH}$ conditions. To produce different positional isomers, the PEGylation of glucagon was also performed at $\mathrm{pH} 9$ and 7. For comparison, the deconvoluted mass spectrum with a post-column addition of DEMA and mass spectrum after ISF for $20 \mathrm{kDa}$ PEGylated glucagon prepared at $\mathrm{pH} 7$ is shown in Figure $3 \mathrm{~b}$ and $\mathrm{d}$, respectively. They are similar to the results (Figure $3 \mathrm{a}$ and c) of the $20 \mathrm{kDa}$ PEGylated glucagon prepared at $\mathrm{pH} 10$. As shown in Figure 4 , the decrease of the reaction $\mathrm{pH}$ led to a significant change in the tandem mass spectra (the precursor ions are always $m / z$ 1367.3). For example, due to the PEGylation, doubly charged $\mathrm{y}_{20}$ ions were observed to be fully shifted from 1261.1 to 1569.3 for the $\mathrm{pH} 10$ produced products. When the reaction was conducted at $\mathrm{pH}$ 9, the peak at 1569.3 became slightly weaker, and a minor peak appeared at 1261.1 indicating the presence of a small portion of unPEGylated $\mathrm{y}_{20}$ ions. Conducting the PEGylation reaction at $\mathrm{pH} 7$ produced an increase in unPEGylated $\mathrm{y}_{20}$ ions compared to the ions for PEGylated species. Thus, the ratio between PEGylated and unPEGylated $\mathrm{y}_{20}$ ions became smaller as the conjugation was carried out under lower $\mathrm{pH}$ conditions, indicating the presence of other positional iso-

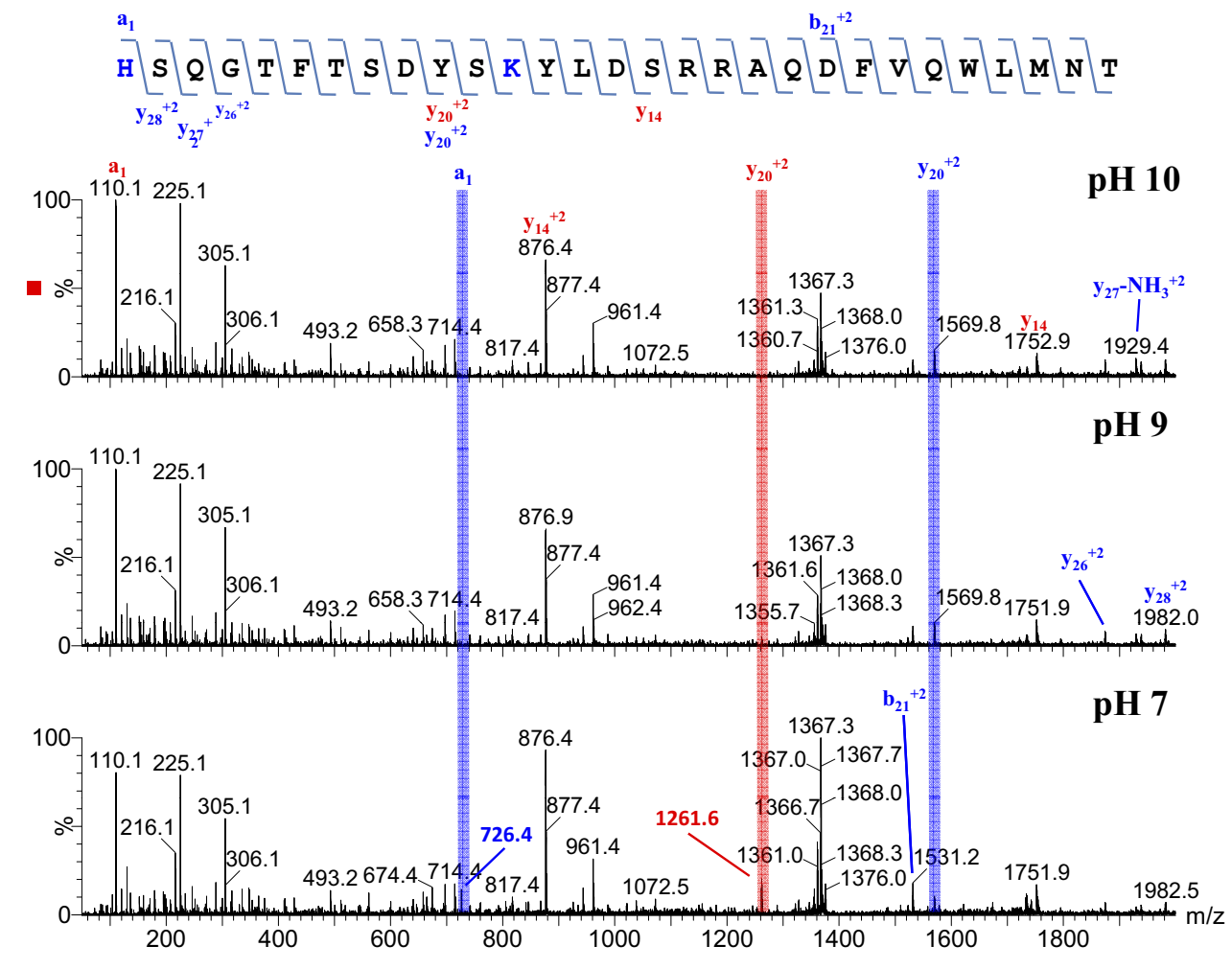

Figure 4. MS/MS spectra of $20 \mathrm{kDa}$ PEGylated glucagon after ISF. The $m / z(z=3)$ of the precursor ion is 1367.3 (glucagon + a small PEG fragment). Top, center and bottom panels represent spectra of PEGylated glucagon prepared at $\mathrm{pH} 10,9$, and 7, and the interpretation of the product ion spectrum is summarized at the top of the spectrum (the detailed MS/MS data assignment is provided in Table 2 in the supplemental materials). The red and blue bars represent the ions without and with PEG attachment, respectively. It should be noted that the different precursor ions for 5 and $20 \mathrm{kDa}$ PEGylated glucagon were selected for MS/MS. The main reasons were (1) further confirming that the small PEG fragment attached is stable during CID, and (2) increasing the MS/MS spectral intensity. 
mers of PEGylated glucagon. A second PEGylation site can be easily identified as the N-terminal His residue based on the clear observation of PEGylated $a_{1}$ ions (shift from $\mathrm{m} / \mathrm{z} 110.1$ to 726.4 ) for the $\mathrm{pH} 7$ products. The other PEGylated ions, a2, b2, b3, b5, b6, and b9, were also observed (see the supplemental tables, which can be found in the electronic version of this article). Taken together, these findings show that our methodology can distinguish different PEGylation sites without any prior separation of positional isomers.

\section{Twenty kDa PEGylated IgG4 Antibody}

Currently marketed PEGylated products are not only composed of small proteins, but also enzymes, oligonucleotides, and antibody fragments [29]. To fully evaluate the capability of our methodology, PEGylated partially reduced IgG4 light chain was also selected as a model compound. To prepare this product, an IgG4 antibody was first treated with DTT under nondenaturing conditions, which only reduced inter-chain disulfide bonds, and produced one unpaired Cys residue in the light chain and three in the heavy chain [30]. The partially-reduced antibody was next incubated with $20 \mathrm{kDa}$ mPEG-MAL, a PEG derivative that selectively reacts with thiol groups of Cys residues, successfully converting the partially reduced light chain to a mono-PEGylated product, as shown in Figure 5a.

Since IgG4 light chain contains a greater number of amino acids than glucagon, the ISF treatment of PEGylated IgG4 light chain yielded more complex mass spectra (Figure 5b). In the 1400-2400 (m/z) range, PEGylated IgG4 light chain fragments were distributed in six different charge states that could be easily deduced to +10 to +15 based on the distance between two adjacent fragment ions having the same charge. To simplify the final data analysis, these mass spectra were deconvoluted as shown in Figure 5c, which revealed that ISF fragments of PEGylated light chain shared a common structure consisting of light chain, a $208 \mathrm{Da}$ linker, and a few oxyethylene units.

Light chain has a molecular mass too large for direct top-down PEGylation site mapping, so Lys-C digestion was employed to reduce the size of PEGylated products before CID MS/MS. In addition, Lys-C digestion can concentrate the ions that distributed in the six different charge states and increase the signal intensity of final tandem mass spectra. Based on enzyme specificity and accurate mass, the constant region fragment 101-107

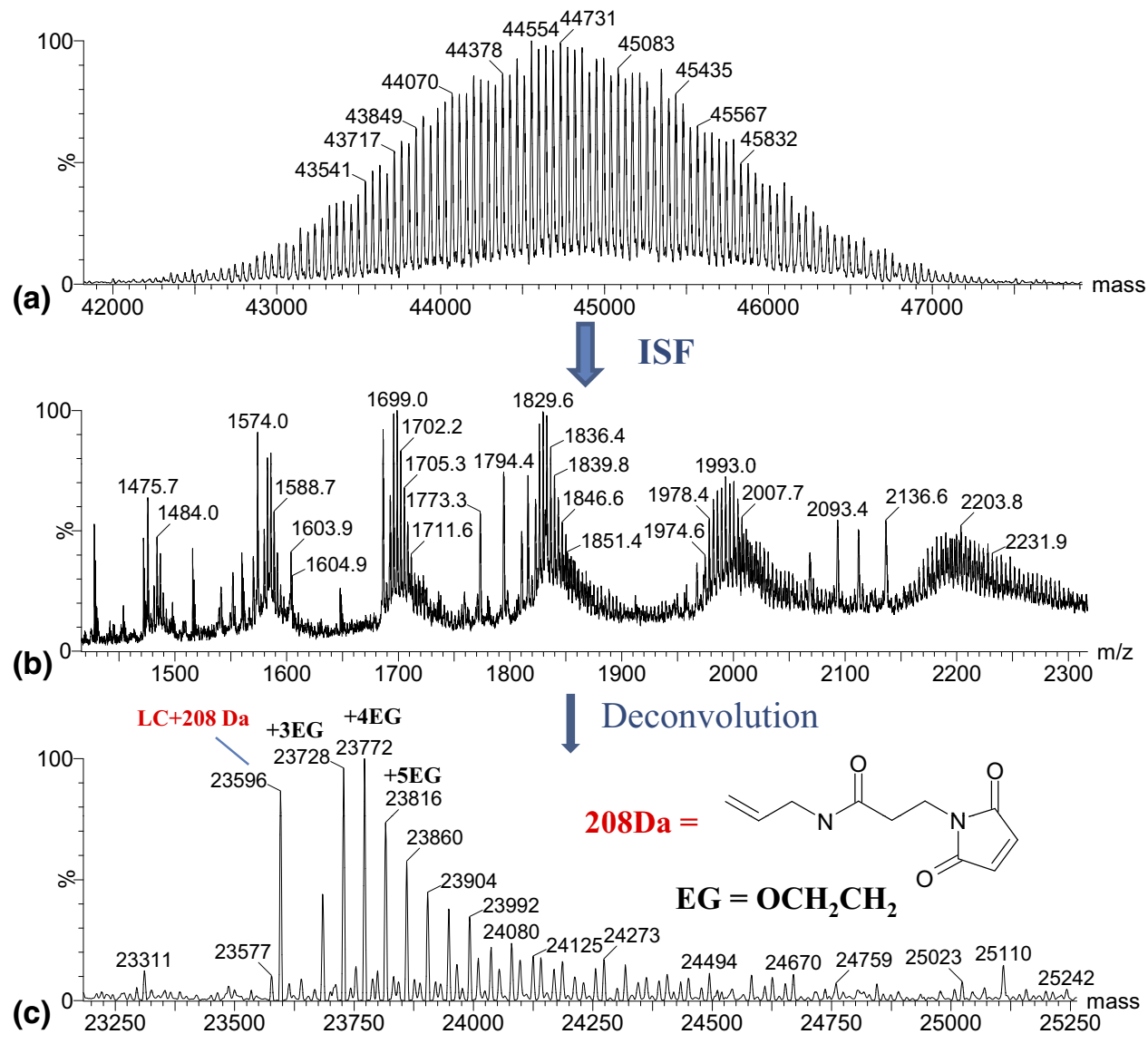

Figure 5. Deconvoluted mass spectrum (a) of $20 \mathrm{kDa}$ PEGylated light chain with a post-column addition of DEMA, mass spectrum (b) and deconvoluted mass spectrum (c) of $20 \mathrm{kDa}$ PEGylated light chain after ISF. 


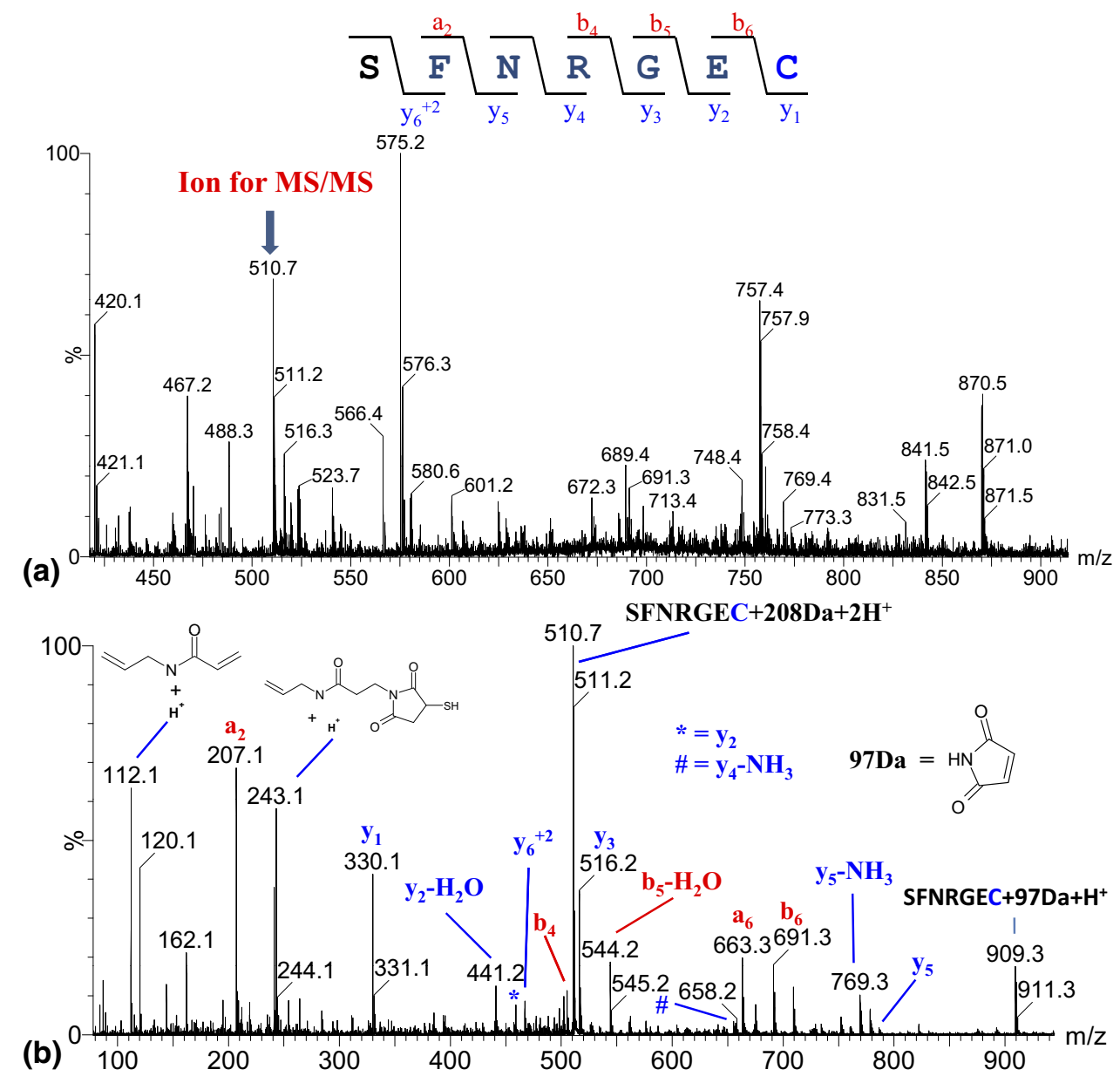

Figure 6. Mass spectrum (a) of PEGylated Lys-C peptides of PEGylated partially reduced IgG4 antibody obtained after ISF and subtracting the background (free PEG peak), and MS/MS spectrum (b) of the precursor ion at $\mathrm{m} / \mathrm{z} 510.7$ (double ion of the peptide SFNRGEC plus a 208 Da linker). The interpretation of the product ion spectrum is summarized at the top of the spectrum (The detailed MS/MS data assignment is provided in Table 3 in the supplemental materials).

(SFNRGEC, 811.9 Da) was found to be PEGylated. According to previous ISF results for PEGylated light chain, the shortest attachment left after the fragmentation is a $208 \mathrm{Da}$ linker, so the shortest PEGylated fragment generated from the combination of Lys-C treatment and ISF is the peptide SFNRGEC plus the linker. Figure 6a is a mass spectrum of PEGylated Lys-C peptides of PEGylated partially reduced IgG4 after ISF and subtracting the background (free PEG peak). The expected double ion (at $\mathrm{m} / \mathrm{z} 510.7$ ) of the peptide SFNRGEC plus $208 \mathrm{Da}$ linker is one major ion. We selected this fragment's doubly charged ions at 510.7 $(\mathrm{m} / \mathrm{z})$ as the precursor ions to perform CID MS/MS. As shown in Figure 6b, all y ions were found to contain the linker, while the $b$ ions were intact, indicating that the PEGylation site is in the constant region and located at the C-terminal Cys of IgG light chain. These results show that our methodology, combined with selective enzyme digestion, can be successfully applied to characterize PEGylated protein products with large molecular mass.

\section{Conclusions}

Elucidation of PEGylation sites is a significant challenge for the characterization of PEGylated peptide and protein products. Here, we described an approach using the combination of ISF and conventional CID MS/MS and found that the small PEG fragment attachment generated by ISF was stable during the CID activation. The experiments with PEGylated glucagon and IgG4 light chain demonstrated that the methodology is suitable for PEGylation site mapping of various PEGylated products without many of the complexities associated with previously described procedures. Furthermore, the application of our methodology to a mixture of different positional isomers successfully distinguishes the distinct PEGylation sites without any prior separation requirement.

\section{Acknowledgments}

The authors thank Andrew Carr for providing $5 \mathrm{kDa}$ PEGylated glucagon. 


\section{Appendix A Supplementary Material}

Supplementary material associated with this article may be found in the online version at doi:10.1016/ j.jasms.2010.01.011.

\section{References}

1. Walsh, G. Biopharmaceutical Benchmarks. Nat. Biotechnol. 2000, 18, 831-833.

2. Bruckdorfer, T.; Marder, O.; Albericio, F. From Production of Peptides in Milligram Amounts for Research to Multi-Ton Quantities for Drugs of the Future. Curr. Pharm. Biotechnol. 2004, 5, 29-43.

3. Sinclair, A. M.; Elliott, S. Glycoengineering: The Effect of Glycosylation on the Properties of Therapeutic Proteins. J. Pharm. Sci. 2005, 94, 1626-1635.

4. Wong, K.; Cleland, L. G.; Poznansky, M. J. Enhanced Anti-Inflammatory Effect and Reduced Immunogenicity of Bovine Liver Superoxide Dismutase by Conjugation with Homologous Albumin. Agents Actions 1980, 10, 231-239.

5. Syed, S.; Schuyler, P. D.; Kulczycky, M.; Sheffield, W. P. Potent Antithrombin Activity and Delayed Clearance from the Circulation Characterize Recombinant Hirudin Genetically Fused to Albumin. Blood 1997, 89, 3243-3252.

6. Lyczak, J. B.; Morrison, S. L. Biological and Pharmacokinetic Properties of a Novel Immunoglobulin-CD4 Fusion Protein. Arch. Virol. 1994, 139, 189-196.

7. Abuchowski, A.; McCoy, J. R.; Palczuk, N. C.; van Es, T.; Davis, F. F. Effect of Covalent Attachment of Polyethylene Glycol on Immunogenicity and Circulating Life of Bovine Liver Catalase. J. Biol. Chem. 1977, 252, 3582-3586.

8. Davis, F. F. The Origin of PEGnology. Adv. Drug Deliv. Rev. 2002, 54, 457-458

9. Harris, J. M.; Chess, R. B. Effect of PEGylation on Pharmaceuticals. Nat. Rev. Drug Discov. 2003, 2, 214-221.

10. Veronese, F. M.; Harris, J. M. Introduction and Overview of Peptide and Protein PEGylation. Adv. Drug Deliv. Rev. 2002, 54, 453-456.

11. Veronese, F. M.; Mero, A. The Impact of PEGylation on Biological Therapies. BioDrugs 2008, 22, 315-329.

12. Lee, K. C.; Moon, S. C.; Park, M. O.; Lee, J. T.; Na, D. H.; Yoo, S. D.; Lee, H. S.; DeLuca, P. P. Isolation, Characterization, and Stability of Positional Isomers of Mono-PEGylated Salmon Calcitonins. Pharm. Res. $1999,16,813-818$

13. Na, D. H.; Youn, Y. S.; Lee, K. C. Optimization of the PEGylation Process of a Peptide by Monitoring with Matrix-Assisted Laser Desorption/ Ionization Time-of-Flight Mass Spectrometry. Rapid Commun. Mass Spectrom. 2003, 17, 2241-2244.

14. Foser, S.; Schacher, A.; Weyer, K. A.; Brugger, D.; Dietel, E.; Marti, S. Schreitmuller, T. Isolation, Structural Characterization, and Antiviral
Activity of Positional Isomers of Mono-PEGylated Interferon $\alpha$-2a (PEGASYS). Protein Expr. Purif. 2003, 30, 78-87.

15. Huang, L.; Gough, P. C.; Defelippis, M. R. Characterization of Poly(ethylene glycol) and PEGylated Products by LC/MS with Post-Column Addition of Amines. Anal. Chem. 2009, 81, 567-577.

16. Iafelice, R.; Cristoni, S.; Caccia, D.; Russo, R.; Rossi-Bernardi, L.; Lowe, K. C.; Perrella, M. Identification of the Sites of Deoxyhemoglobin PEGylation. Biochem. J. 2007, 403, 189-196.

17. Roberts, M. J.; Harris, J. M. Attachment of Degradable Poly(ethylene glycol) to Proteins Has the Potential to Increase Therapeutic Efficacy. J. Pharm. Sci. 1998, 87, 1440-1445.

18. Yoo, C.; Suckau, D.; Sauerland, V.; Ronk, M.; Ma, M. Toward Top-Down Determination of PEGylation Site Using MALDI In-Source Decay MS Analysis. J. Am. Soc. Mass Spectrom. 2009, 20, 326-333.

19. Veronese, F. M. Peptide and Protein PEGylation: A Review of Problems and Solutions. Biomaterials 2001, 22, 405-417.

20. Clark, R.; Olson, K.; Fuh, G.; Marian, M.; Mortensen, D.; Teshima, G.; Chang, S.; Chu, H.; Mukku, V.; Canova-Davis, E.; Somers, T.; Cronin M.; Winkler, M.; Wells, J. A. Long-Acting Growth Hormones Produced by Conjugation with Polyethylene Glycol. J. Biol. Chem. 1996, 271, 21969-21977.

21. Veronese, F. M.; Sacca, B.; de Laureto, P.; Sergi, M.; Caliceti, P.; Schiavon, O.; Orsolini, P. New PEGs for Peptide and Protein Modification, Suitable for Identification of the PEGylation Site. Bioconj. Chem. 2001, 12, 62-70.

22. Vestling, M. M.; Murphy, C. M.; Keller, D. A.; Fenselau, C.; Dedinas, J Ladd, D. L.; Olsen, M. A. A Strategy for Characterization of Polyethylene Glycol-Derivatized Proteins. A Mass Spectrometric Analysis of the Attachment Sites in Polyethylene Glycol-Derivatized Superoxide Dismutase. Drug Metab. Dispos. 1993, 21, 911-917.

23. Lee, H.; Park, T. G. A Novel Method for Identifying PEGylation Sites of Protein Using Biotinylated PEG Derivatives. J. Pharm. Sci. 2003, 92, 97-103.

24. Wang, Y. S.; Youngster, S.; Bausch, J.; Zhang, R.; McNemar, C.; Wyss, D. F. Identification of the Major Positional Isomer of PEGylated Interferon $\alpha$-2b. Biochemistry 2000, 39, 10634-10640.

25. Salmaso, S.; Bersani, S.; Scomparin, A.; Mastrotto, F.; Scherpfer, R.; Tonon, G.; Caliceti, P. Tailored PEG for rh-G-CSF Analogue Site-Specific Conjugation. Bioconjug. Chem. 2009, 20, 1179-1185.

26. Roberts, M. J. Bentley, M. D. Harris, J. M. Chemistry for Peptide and Protein PEGylation. Adv. Drug Delivery Rev. 2002, 54, 459-476.

27. Carr, A.; Second, T.; Cummins, R.; Viner, R.; Huang, L. Characterization of PEGylated Peptides and Site Localization of Attachment with High Resolution ETD Mass Spectrometry. Proceedings of the 57th ASMS Conference on Mass Spectrometry and Allied Topics; Philadelphia, PA, May, 2009

28. Jorgensen, K. E.; Moller, J. V. Use of Flexible Polymers as Probes of Glomerular Pore Size. Am. J. Physiol. 1979, 236, F103-111.

29. Kang, J. S.; Deluca, P. P.; Lee, K. C. Emerging PEGylated Drugs. Expert Opin. Emerg. Drugs 2009, 14, 363-380.

30. Huang, L.; Mitchell, C. E. High-Throughput LC/MS Methodology for $\alpha(1 \rightarrow 3)$ Gal Determination of Recombinant Monoclonal Antibodies. In Therapeutic Protein, Methods, and Protocols, Smales, C. M.; James, D. C., Eds.; Humana Press: Totowa, NJ, 2005; p. 411-419. 